\title{
cmaJOPEN
}

\section{A comparison of 3 frailty measures and adverse outcomes in the intake home care population: a retrospective cohort study}

\author{
Chi-Ling Joanna Sinn PhD, George Heckman MD MSc, Jeffrey W. Poss PhD, Graziano Onder MD PhD, \\ Davide Liborio Vetrano MD PhD, John Hirdes PhD
}

\section{Abstract}

Background: In Ontario, Canada, nearly all home care patients are assessed with a brief clinical assessment (interRAI Contact Assessment [interRAI CA]) on admission. Our objective was to compare 3 frailty measures that can be operationalized using the interRAI CA.

Methods: We conducted a retrospective cohort study using linked patient-level assessment and administrative data for all Ontario adult ( $\geq 18 \mathrm{yr}$ ) home care patients assessed with the interRAI CA in 2014. We employed multivariable logistic models to compare the Changes in Health, End-stage disease and Signs and Symptoms Scale for the Contact Assessment (CHESS-CA), Assessment Urgency Algorithm (AUA) and the Frailty Index for the Contact Assessment (FI-CA) that was created for this study. Our outcomes of interest were death, hospital admission and emergency department visits within 90 days, and assessor-rated need for comprehensive geriatric assessment (CGA).

Results: In 2014, there were 228679 unique adult home care patients in Ontario assessed with the interRAI CA. Controlling for age, sex and health region, being in a higher frailty level defined by any measure increased the likelihood of experiencing adverse outcomes. Among all assessments, CHESS-CA was best suited for predicting death and hospital admission, and either AUA or FI-CA for predicting perceived need for CGA. Previous emergency department visits were more predictive of future visits than frailty. Model fit was independent of whether the assessment was completed over the phone or in person.

Interpretation: Frailty measures from the interRAI CA identified patients at higher risk for death, hospital admission and perceived need for CGA. In jurisdictions where the CHESS-CA and AUA are already built into the electronic home care platform, such as Ontario, patients identified as high risk should be prioritized for proactive referral and care planning, and may benefit from greater involvement of primary care and other health professionals in the circle of care.

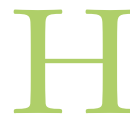
ome care serves people of all ages and with broad complexities of needs. Home care services may be used to support patient recovery after hospital discharge, or to help patients cope with life-limiting illness or manage chronic conditions, disabilities or mental illnesses, among other functions. ${ }^{1}$ The availability of communitybased care options - in conjunction with substantial contributions by unpaid caregivers - makes it possible to support people with care needs at home, thus reducing demand on high-cost hospital and institutional beds..$^{2-4}$ Ultimately, the goal of home care is to meet the patient's needs so that they require the same (or lesser) level of care over time; however, frailty has considerable influence on whether this goal can be achieved.

Frailty describes a state of vulnerability as a result of agerelated declines in multiple physiologic systems. ${ }^{5}$ When faced with a stressor, a frail person is more likely to experience sudden health state changes and is less likely to return to their premorbid health state. In this state of poor recovery, frailty increases the risk of adverse outcomes, such as increased dependency and disability, high health service use and death. ${ }^{5-7}$ Emerging evidence about coronavirus disease 2019 (COVID-19) suggests that older people and those with comorbidities (especially of the heart, lung, kidney and liver)

Competing interests: None declared.

This article has been peer reviewed.

Correspondence to: Chi-Ling Sinn, cjsinn@uwaterloo.ca

CMAJ Open 2020. DOI:10.9778/cmajo.20200083 
are at the highest risk of severe illness and death, and it is thought that these characteristics may be indicative of underlying frailty. ${ }^{8-11}$ It is clear that frailty must be identified early to guide comprehensive care planning to avoid preventable disability and death.

In Ontario, most adult home care patients are assessed with the interRAI Contact Assessment (interRAI CA; about 50 clinical items) upon admission to home care services. ${ }^{12}$ About one-third of patients are expected to require services for longer than 2 months and are followed up with the more comprehensive interRAI Home Care assessment (interRAI HC [formerly, RAI-HC]; about 250 clinical items). ${ }^{13}$ The interRAI HC produces several frailty measures, including the interRAI Home Care Frailty Scale,${ }^{14}$ Changes in Health, End-stage disease and Signs and Symptoms (CHESS) Scale, ${ }^{15}$ and a number of frailty indices..$^{16,17}$

A frailty measure that would apply to all home care patients on admission is needed to support referral and care planning decisions. In this paper, we compare 3 frailty measures that can be operationalized using the interRAI CA based on their relative ability to predict adverse outcomes and assessor-rated need for comprehensive geriatric assessment (CGA).

\section{Methods}

\section{Study design}

We conducted a retrospective cohort study involving adult home care patients in Ontario using linked patient-level assessment and administrative data. The following databases held by the Canadian Institute for Health Information (CIHI) were used in this study: Home Care Reporting System (HCRS) for home care referrals and interRAI CA assessments, Discharge Abstract Database (DAD) for hospital admissions and National Ambulatory Care Reporting System (NACRS) for emergency department visits. Data were linked by $\mathrm{CIHI}$ and provided in de-identified form to the University of Waterloo.

\section{Setting}

In Ontario, publicly funded home care is available across the province, although home care programs and services are locally funded, planned and managed by health regions. Any individual can apply for home care services. Care coordinators who are health professionals (often nurses) employed by the health regions receive these referrals and assess the person's needs using the interRAI CA. Existing home care patients returning from hospital are also assessed with the interRAI CA. Care coordinators can complete the assessment by drawing on multiple information sources, such as speaking with the patient or their family (in person or by phone), speaking with other health professionals or care providers and referring to health records or written notes. Once the assessment is complete, standard algorithms are automatically calculated and shown on the patient's dashboard. Previous studies have shown the validity and reliability of the interRAI suite of instruments. ${ }^{18-22}$

\section{Participants}

The study population comprised unique adult ( $\geq 18 \mathrm{yr}$ ) home care patients in Ontario assessed with the interRAI CA between Jan. 1 and Dec. 31, 2014. We selected the most recent interRAI CA if a patient was assessed more than once within the calendar year. Each assessment was linked with the patient's home care referral that was open at the time of assessment and DAD and NACRS records, if available.

\section{Frailty measures}

We selected 3 frailty measures that can be operationalized using the interRAI CA. The assessment items used to construct each of the measures are listed in Appendix 1 (available at www.cmajopen.ca/content/8/4/E796/suppl/DC1).

1) Changes in Health, End-stage disease and Signs and Symptoms Scale for the Contact Assessment (CHESSCA): CHESS-CA is a summary measure of health instability that ranges from 0 (no health instability) to 5 (very high health instability). Although not explicitly named as a frailty scale, it taps key concepts related to frailty (e.g., functional decline) and the CHESS scale from the interRAI HC has been shown to predict health service use, caregiver distress and death among long-stay home care patients. ${ }^{15,16,23}$

2) Assessment Urgency Algorithm (AUA): AUA is a riskscreening algorithm that ranges from 1 to 6 . Higher AUA levels indicate greater need and priority for a comprehensive follow-up assessment. ${ }^{12}$ Patients without cognitive or functional impairment are classified into levels 1 through 3 based on shortness of breath, unstable patterns and poor self-rated health. Patients with any impairment are classified into levels 4 through 6 based on signs of poor personal or family coping.

3) Frailty Index for the Contact Assessment (FI-CA): A frailty index counts a person's health deficits across the body's physiologic systems and is expressed as a ratio ranging from 0 to $1 .^{5}$ Frailty indices are widely used in primary and community settings, so the index used in this study was considered to be the reference standard. ${ }^{24-26}$ In contrast to CHESS-CA and AUA, which are standard interRAI algorithms, FI-CA was created for this study by adapting the Emergency Department Frailty Index (FI-ED), a frailty index that was developed for the Emergency Department version of the Contact Assessment and validated in a multinational cohort. ${ }^{27}$ Both the FI-CA and FI-ED count up to 24 deficits, of which 18 deficits are identical in measurement. An additional 6 deficits were added into the FI-CA that satisfy the 5 criteria for creating frailty indices as outlined by Searle and colleagues and are also present in the interRAI Home Care Frailty Scale. ${ }^{14,28}$

\section{Outcomes}

The following outcomes were extracted if they occurred within 90 days of the assessment date: death based on discharge codes recorded in HCRS, DAD or NACRS; emergency department 
visits based on any matching record in NACRS; and nonelective hospital admissions based on any matching record in DAD. An item in the interRAI CA about the assessor's rating of the patient's need for CGA (yes v. no) was used as an additional dependent variable.

\section{Statistical analysis}

Four logistic models were developed for each outcome: first, a base model that included age, sex and health region, and then a model for each frailty measure added to the base covariates. For sensitivity analyses, all models were re-run using alternately sampled or restricted datasets. To enable comparison of FI-CA with the other ordinal measures, we created 6 FI-CA categories that were informed by cut-off points reported in the literature..$^{29,30}$ Previous studies have generally adopted the following thresholds: $\mathrm{FI}<0.10$ (less fit), $\mathrm{FI}<0.21$ (least fit), $\mathrm{FI}<0.45$ (frail) and $\mathrm{FI} \geq 0.45$ (most frail). Other studies propose defining the thresholds based on the sample's FI distribution. ${ }^{29,30}$ Brousseau and colleagues ${ }^{27}$ suggest defining the most frail groups using the top 25 th and 10 th percentile scores. Calculation of the area under the curve (AUC) statistics permitted comparison of model goodness of fit. Sociodemographic, health service use and clinical characteristics (used in at least 1 of the frailty measures) are reported in frequency tables. Age-related differences (age $\geq 65 \mathrm{yr}$ v. $<65 \mathrm{yr}$ ) were calculated using $\chi^{2}$ tests. Spearman rank order correlations were calculated between frailty measures. We used SAS software version 9.4 for all analyses (SAS Institute Inc.).

\section{Ethics approval}

Ethics approval for this research, including the procedures followed to protect privacy and confidentiality, was obtained from the Office of Research Ethics at the University of Waterloo (ORE no. 18228).

\section{Results}

In 2014, there were 228679 unique adult home care patients in Ontario assessed with the interRAI CA. Table 1 summarizes the sociodemographic and health service use characteristics of the intake home care population. The mean age was 71.8 (standard deviation 15.5 ) years. Overall, $71.8 \%$ of patients were 65 years and older, $57.4 \%$ were female, $29.3 \%$ lived alone and $96.0 \%$ identified a primary informal caregiver. Recent health service use was high: $60.7 \%$ of patients had been admitted to hospital in the last 90 days, and $32.6 \%$ had visited the emergency department without being admitted to hospital. Similar volumes of patients were assessed in the hospital and community setting. More than half $(54.6 \%)$ of interRAI CAs were done exclusively over the phone (i.e., without in-person assessment).

Table 2 summarizes the clinical characteristics. Patients who were 65 years and older were significantly more likely to have cognitive and functional impairments, shortness of breath, unstable health patterns and recent falls, whereas patients younger than 65 years reported higher rates of poor self-rated health, sad or depressed mood, and pain. Patients younger than 65 years were also significantly more likely to have a cancer diagnosis.

Figure 1 shows the distribution of the intake home care population across frailty measures. The distribution varied by the measure used: the 2 highest risk levels accounted for $6.7 \%$ (CHESS-CA), $36.5 \%$ (AUA) or $26.5 \%$ (FI-CA) of the total population. About half (47.5\%) of the population was in the 2 lowest CHESS-CA levels. CHESS-CA tended to classify patients with a cancer diagnosis higher than other measures, whereas AUA tended to classify patients with congestive heart failure or Alzheimer disease or related dementia higher than other measures. There was moderate to substantial overlap among the measures. The highest correlation coefficient was observed between AUA and FI-CA (continuous; 0.806, $p<$ 0.001). Moderate levels of correlation were observed between CHESS-CA and FI-CA (continuous; 0.622, $p<0.001$ ) and CHESS-CA and AUA $(0.407, p<0.001)$.

Overall, $7.3 \%$ (16 778) of patients died, 16.6\% (38 055) were admitted to hospital and $44.4 \%$ (101 526) went to the emergency department within 90 days of the assessment date. Half of the population $(114027$; 49.9\%) was rated by the assessor as requiring CGA.

Tables 3, 4, 5 and 6 summarize the multivariable logistic models for each study outcome and frailty measure. The CHESS-CA models produced the highest AUC statistics for death (0.768) and hospital admission (0.617). A similar AUC statistic was observed for FI-CA and hospital admission (0.609). Notably, none of the frailty measures were able to improve the prediction of future emergency department visits substantially above the base model. Instead, previous emergency department visits produced the highest AUC statistic for future emergency department visits (0.581). The FI-CA model produced the highest AUC statistic for perceived need for CGA (0.796).

Older age and male sex were consistently associated with greater odds of adverse outcomes, except for negative associations observed between age and emergency department visits and between male sex and need for CGA. In some cases, the odds ratios associated with living in a certain health region were at least as large as the odds ratios for other covariates and even moderate frailty levels. Model fit did not vary significantly when the results were restricted to assessments completed exclusively over the phone. Additional sensitivity analyses showed that the overall results did not change whether a patient's most recent assessment or a randomly selected assessment was used.

To assist with clinical interpretation, Tables 7, 8 and 9 illustrate the observed proportions of each study outcome (where discrimination was possible) by age, sex and frailty measure.

\section{Interpretation}

Among Ontario home care patients assessed with the interRAI CA, $7.3 \%$ died, $16.6 \%$ were admitted to hospital and $44.4 \%$ went to the emergency department within 90 days. Despite a relatively short observation period, these adverse 


\begin{tabular}{|c|c|c|c|}
\hline \multirow[b]{2}{*}{ Characteristic } & \multicolumn{3}{|c|}{$\%$ (no.) of patients } \\
\hline & $\begin{array}{l}\text { All patients } \\
n=228679\end{array}$ & $\begin{array}{c}\text { Patients aged }<65 \mathrm{yr} \\
n=64543\end{array}$ & $\begin{array}{c}\text { Patients aged } \\
\geq 65 \mathrm{yr} \\
n=164136\end{array}$ \\
\hline \multicolumn{4}{|l|}{ Age group, $\mathrm{yr}^{*}$} \\
\hline $18-49$ & $8.8(20209)$ & 31.3 (20 209) & - \\
\hline $50-59$ & $11.3(25798)$ & $40.0(25798)$ & - \\
\hline $60-69$ & $18.2(41665)$ & $28.7(18536)$ & $14.1(23129)$ \\
\hline $70-79$ & $24.4(55755)$ & - & $34.0(55755)$ \\
\hline $80-89$ & $28.4(65047)$ & - & $39.6(65047)$ \\
\hline$\geq 90$ & $8.8(20$ 205) & - & $12.3(20205)$ \\
\hline \multicolumn{4}{|l|}{ Sex $^{*}$} \\
\hline Female & $57.4(131217)$ & $54.1(34931)$ & $58.6(96286)$ \\
\hline Male & $42.6(97462)$ & $45.9(29612)$ & $41.3(67850)$ \\
\hline \multicolumn{4}{|l|}{ Living arrangement* } \\
\hline Alone & $29.3(66944)$ & 21.5 (13 919) & $32.3(53025)$ \\
\hline With spouse or partner & $46.4(106$ 142) & $53.0(34227)$ & 43.8 (71 915) \\
\hline With other family & $16.0(36562)$ & $18.3(11862)$ & $15.0(24700)$ \\
\hline With nonrelatives & $8.3(19031)$ & $7.0(4535)$ & $8.8(14496)$ \\
\hline \multicolumn{4}{|l|}{ Primary informal caregiver present ${ }^{\star}$} \\
\hline Yes, spouse or partner & 45.7 (104 492) & $54.3(35089)$ & $42.3(69403)$ \\
\hline Yes, other relationship & $50.3(115078)$ & $39.5(25527)$ & $54.5(89551)$ \\
\hline No informal caregiver & $4.0(9109)$ & $6.1(3927)$ & $3.1(5182)$ \\
\hline \multicolumn{4}{|l|}{ Disease diagnoses $†$} \\
\hline Congestive heart failure* & $7.2(16439)$ & $2.4(1565)$ & $9.0(14874)$ \\
\hline $\begin{array}{l}\text { Chronic obstructive pulmonary } \\
\text { disease* }^{*}\end{array}$ & $8.7(19816)$ & $5.2(3389)$ & $10.0(16427)$ \\
\hline $\begin{array}{l}\text { Alzheimer disease or related } \\
\text { dementia* }^{*}\end{array}$ & $7.0(15984)$ & $0.6(355)$ & $9.5(15629)$ \\
\hline Cancer $^{\star}$ & $14.3(32800)$ & $16.6(10715)$ & $13.4(22085)$ \\
\hline \multicolumn{4}{|l|}{ Health service use in last $90 d$} \\
\hline ED visit(s), without hospital admission & $32.6(74$ 491) & $32.8(21$ 173) & 32.5 (53 318) \\
\hline Hospital admission(s)* & $60.7(138829)$ & $62.3(40$ 237) & $60.0(98592)$ \\
\hline \multicolumn{4}{|l|}{ Patient's location at time of assessment* } \\
\hline ED or hospital inpatient & $48.4(110692)$ & $46.12(29788)$ & $49.3(80904)$ \\
\hline Community & $46.7(106685)$ & $46.5(30013)$ & $46.7(76672)$ \\
\hline Other & $4.9(11302)$ & $7.3(4742)$ & $4.0(6560)$ \\
\hline \multicolumn{4}{|l|}{ Type of communication at intake* } \\
\hline Phone only & 54.6 (124 939) & $57.1(36860)$ & $53.6(88079)$ \\
\hline Other methods $\ddagger$ & $45.4(103740)$ & $42.9(27683)$ & $46.3(76057)$ \\
\hline
\end{tabular}




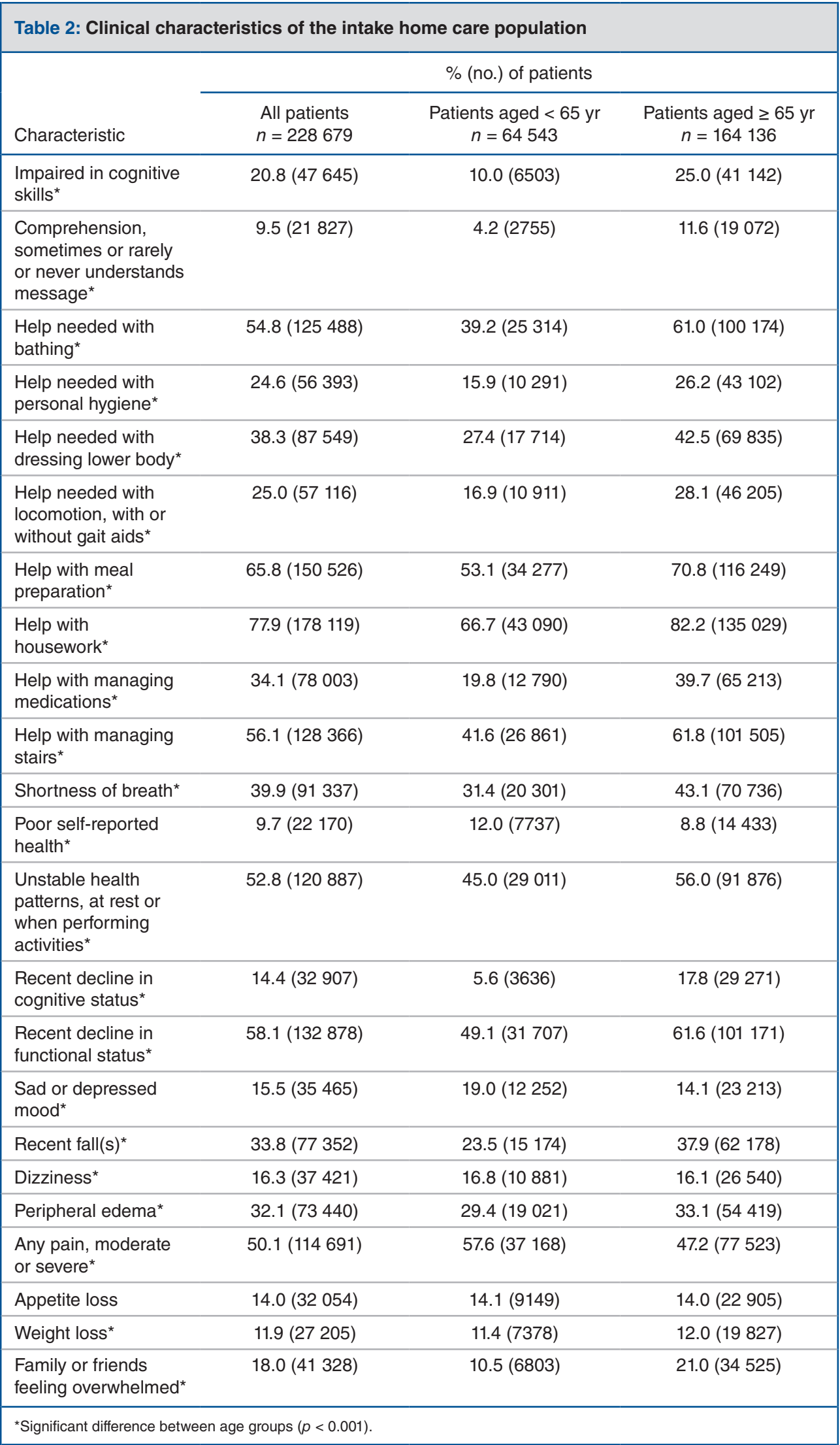




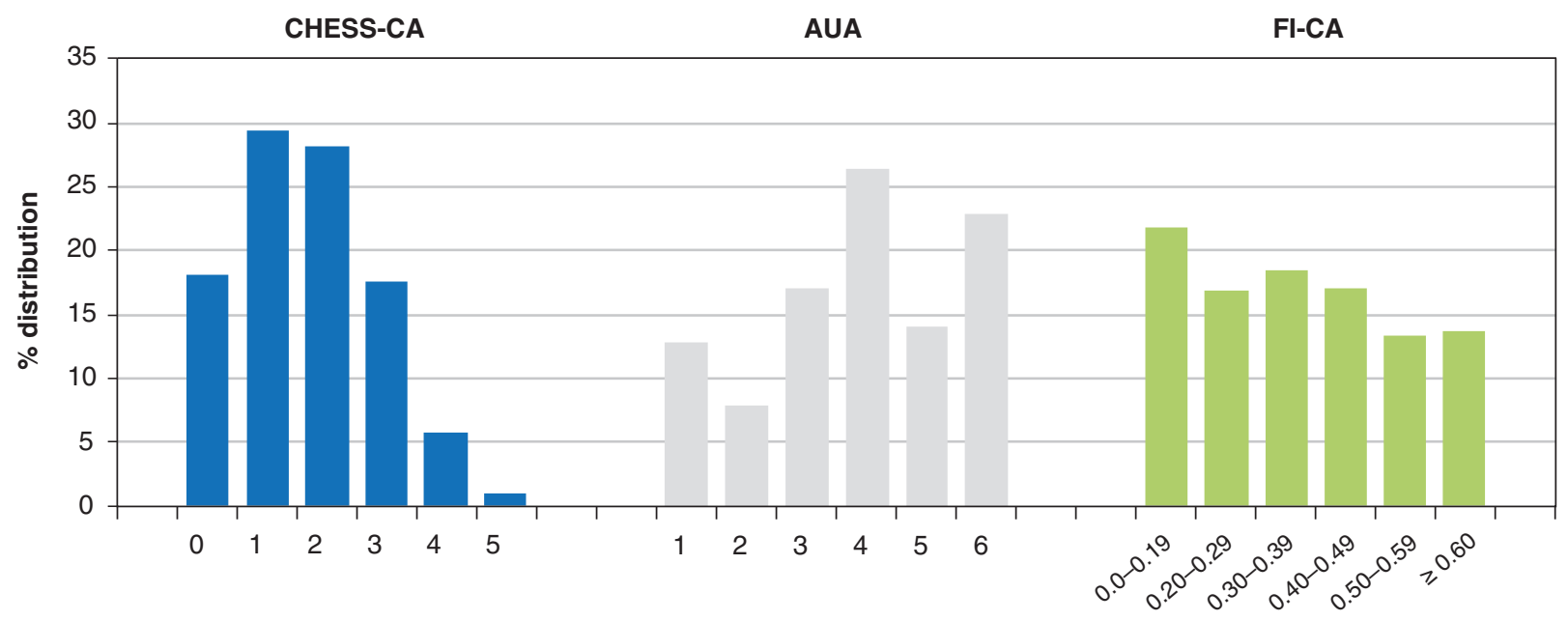

Frailty index, score

Figure 1: Percent distribution of intake home care population across frailty measures. Note: AUA = Assessment Urgency Algorithm; CHESS-CA = Changes in Health, End-stage disease and Signs and Symptoms Scale for the Contact Assessment; FI-CA = Frailty Index for the Contact Assessment.

outcomes affected a considerable proportion of the intake home care population and illustrate the need to consider comprehensive, proactive care planning for the broader home care population (i.e., not just long-stay patients). This study shows the potential utility of a frailty algorithm embedded in the intake assessment process for identifying vulnerable individuals early in the home care episode.

We believe that this study provides additional evidence for the validity of all 3 measures (CHESS-CA, AUA, FI-CA) and extends their application to all home care clients, regardless of the short- or long-term nature of required services. Controlling for age, sex and health region, being in a higher frailty level defined by any measure consistently increased the likelihood of experiencing adverse outcomes. Among all assessments, CHESS-CA was best suited for predicting death and admission to hospital, and either AUA or FI-CA for predicting perceived need for CGA. Often, the best model produced only incremental gains in predictive performance above the next best model.

Previous studies have also identified well-performing measures of frailty despite not finding a single measure that outperforms the rest. ${ }^{16,31}$ The AUC statistics observed for death and hospital admission in the CHESS-CA and FI-CA models among this study's intake home care population are strikingly similar to those reported for long-stay patients. Hirdes and colleagues observed an AUC statistic of 0.752 in their CHESS model after adjusting for age, sex and diagnoses. Campitelli and colleagues observed AUC statistics of 0.607 and 0.600 in their CHESS and FI models, respectively, after adjusting for age, sex and comorbidity. ${ }^{15,31}$ The intake home care population is relatively younger and has higher levels of health instability compared to the subset of long-stay patients, yet the frailty measures yielded similar predictive performance. Outside of Ontario, CHESS-CA was strongly associated with home care intensity (i.e., ratio of days with home care visits) after older Italian patients were discharged from hospital to home. ${ }^{32}$ Taken together, our findings add to existing evidence that these frailty measures can be applied across the home care population, and where compatible measures exist across assessment systems, allow for direct comparisons between populations.

Brousseau and colleagues ${ }^{27}$ developed the Emergency Department Frailty Index (FI-ED) out of the interRAI EDContact Assessment and found that the FI-ED was significantly associated with hospital admission, hospital death, prolonged hospital stay, discharge to long-term care and perceived need for CGA. As sister instruments, the interRAI ED-CA and interRAI CA share many of the same clinical items, and thus, there is a $75 \%$ overlap in the items contained in the FI-ED and FI-CA. It is reasonable, therefore, to expect that the results of these 2 studies apply to patient populations and settings using either assessment instrument.

The lack of a strong relation between frailty and emergency department visits is consistent with other studies of community-dwelling older adults. ${ }^{33,34}$ The Detection of Indicators and Vulnerabilities for Emergency department Trips (DIVERT) Scale in the interRAI HC suggests that previous emergency department use, cardiorespiratory symptoms and cardiac conditions are the main drivers..$^{35}$ Similarly, in the present study, having an emergency department visit in the last 90 days was a better predictor of future emergency department visits than any frailty measure. 
Table 3: Adjusted odds ratios of death within 90 days of interRAI CA assessment among the intake home care population

Adjusted OR $(95 \% \mathrm{Cl})^{*}$

\begin{tabular}{|c|c|c|}
\hline Variable & $\begin{array}{l}\text { Base model } \\
\text { (age, sex, health } \\
\text { region only) }\end{array}$ & $\begin{array}{l}\text { CHESS-CA, } \\
\text { adjusted for age, } \\
\text { sex, health region }\end{array}$ \\
\hline \multicolumn{3}{|c|}{ CHESS-CA, score } \\
\hline 0 & & Reference \\
\hline 1 & & $1.67(1.53-1.81)$ \\
\hline 2 & & $3.02(2.80-3.27)$ \\
\hline 3 & & $6.69(6.20-7.23)$ \\
\hline 4 & & $20.38(18.01-22.09)$ \\
\hline 5 & & $63.21(56.59-70.61)$ \\
\hline
\end{tabular}

AUA, score

1

2

3

4

5

6
$A \cup A$,

adjusted for age,

sex, health region
FI-CA,

adjusted for age, sex, health region
FI-CA, score

0-0.19

0.20-0.29

0.30-0.39

0.40-0.49

0.50-0.59

$\geq 0.60$

\section{Reference}

1.86 (1.64-2.11)

4.22 (3.82-4.66)

3.26 (2.96-3.59)

7.57 (6.87-8.33)

$8.24(7.50-9.05)$

Reference

1.71 (1.57-2.85)

2.61 (2.42-2.82)

3.82 (3.55-4.11)

5.81 (5.40-6.25)

11.66 (10.88-12.51)

Age group, yr

\begin{tabular}{lccccc}
\hline $18-49$ & Reference & Reference & Reference & Reference \\
\hline $50-59$ & $1.85(1.68-2.04)$ & $1.48(1.34-1.64)$ & $1.83(1.66-2.03)$ & $1.74(1.57-1.92)$ \\
\hline $60-69$ & $2.40(2.20-2.63)$ & $1.77(1.61-1.94)$ & $2.32(2.12-2.54)$ & $2.10(1.92-2.31)$ \\
\hline $70-79$ & $2.80(2.56-3.06)$ & $1.87(1.71-2.05)$ & $2.43(2.23-2.66)$ & $2.04(1.87-2.23)$ \\
\hline $80-89$ & $3.10(2.84-3.38)$ & $1.93(1.76-2.11)$ & $2.40(2.20-2.62)$ & $1.87(1.71-2.04)$ \\
\hline 90 & $4.46(4.06-4.89)$ & $2.64(2.39-2.90)$ & $3.19(2.90-3.51)$ & $2.29(2.08-2.52)$
\end{tabular}

Sex

Female

\section{Reference}

1.62 (1.57-1.68)

Reference

1.62 (1.57-1.67)

Reference

1.69 (1.63-1.74)

Reference

Health region

Central

Reference

$1.02(0.98-1.07)$

Reference

$1.17(1.12-1.22)$

Reference

$1.18(1.14-1.23)$

$0.96(0.92-1.00)$

$1.00(0.96-1.05)$

\begin{tabular}{|lc}
\hline East & 1.02 \\
\hline North & 0.97 \\
\hline Toronto & 1.02 \\
\hline
\end{tabular}

C statistic for all assessments

$1.02(0.96-1.09)$

$1.07(1.00-1.15)$

$1.14(1.06-1.21)$

0.95 (0.87-1.03)

$0.77(0.71-0.84)$

$1.68(1.63-1.74)$

$0.74(0.68-0.80)$

0.768
0.777

0.610

0.777

0.691

0.693

Reference

$1.28(1.23-1.34)$

$1.00(0.96-1.05)$

$1.20(1.12-1.28)$

$0.86(0.79-0.93)$

C statistic for assessments completed exclusively over the phone

Note: $\mathrm{AUA}=$ Assessment Urgency Algorithm; CHESS-CA = Changes in Health, End-stage disease and Signs and Symptoms Scale for the Contact Assessment; Cl = confidence interval; FI-CA = Frailty Index for the Contact Assessment; interRAI CA = interRAI Contact Assessment; OR = odds ratio.

*Unless stated otherwise. 


\begin{tabular}{|c|c|c|c|c|}
\hline \multirow[b]{2}{*}{ Variable } & \multicolumn{4}{|c|}{ Adjusted OR $(95 \% \mathrm{Cl})^{*}$} \\
\hline & $\begin{array}{l}\text { Base model } \\
\text { (age, sex, health } \\
\text { region only) }\end{array}$ & $\begin{array}{c}\text { CHESS-CA, } \\
\text { adjusted for age, } \\
\text { sex, health region }\end{array}$ & $\begin{array}{c}\text { AUA, } \\
\text { adjusted for age, } \\
\text { sex, health region }\end{array}$ & $\begin{array}{c}\text { FI-CA, } \\
\text { adjusted for age, } \\
\text { sex, health regior }\end{array}$ \\
\hline \multicolumn{5}{|l|}{ CHESS-CA, score } \\
\hline 0 & & Reference & & \\
\hline 1 & & $1.29(1.24-1.34)$ & & \\
\hline 2 & & $2.73(2.67-2.80)$ & & \\
\hline 3 & & $2.54(2.45-2.65)$ & & \\
\hline 4 & & $3.31(3.15-3.48)$ & & \\
\hline 5 & & $2.35(2.12-2.61)$ & & \\
\hline \multicolumn{5}{|l|}{ AUA, score } \\
\hline 1 & & & Reference & \\
\hline 2 & & & $1.52(1.43-1.61)$ & \\
\hline 3 & & & $2.07(1.98-2.17)$ & \\
\hline 4 & & & $1.78(1.70-1.86)$ & \\
\hline 5 & & & $2.40(2.29-2.52)$ & \\
\hline 6 & & & $2.70(2.58-2.83)$ & \\
\hline \multicolumn{5}{|l|}{ FI-CA, score } \\
\hline $0-0.19$ & & & & Reference \\
\hline $0.20-0.29$ & & & & $1.24(1.19-1.29)$ \\
\hline $0.30-0.39$ & & & & $1.52(1.46-1.58)$ \\
\hline $0.40-0.49$ & & & & $1.84(1.77-1.91)$ \\
\hline $0.50-0.59$ & & & & $2.22(2.13-2.31)$ \\
\hline$\geq 0.60$ & & & & $2.70(2.59-2.81)$ \\
\hline \multicolumn{5}{|l|}{ Age group, yr } \\
\hline $18-49$ & Reference & Reference & Reference & Reference \\
\hline $50-59$ & $1.17(1.10-1.23)$ & $1.07(1.01-1.13)$ & $1.15(1.09-1.22)$ & $1.13(1.07-1.19)$ \\
\hline $60-69$ & $1.26(1.20-1.32)$ & $1.11(1.06-1.17)$ & $1.23(1.17-1.30)$ & $1.18(1.12-1.24)$ \\
\hline $70-79$ & $1.40(1.33-1.46)$ & $1.19(1.13-1.24)$ & $1.31(1.25-1.37)$ & $1.22(1.16-1.27)$ \\
\hline $80-89$ & $1.46(1.40-1.53)$ & $1.21(1.15-1.26)$ & $1.31(1.25-1.37)$ & $1.17(1.12-1.23)$ \\
\hline$\geq 90$ & $1.66(1.57-1.75)$ & $1.33(1.26-1.41)$ & $1.43(1.35-1.51)$ & $1.23(1.17-1.30)$ \\
\hline \multicolumn{5}{|l|}{ Sex } \\
\hline Female & Reference & Reference & Reference & Reference \\
\hline Male & $1.36(1.33-1.39)$ & $1.37(1.33-1.40)$ & $1.38(1.35-1.41)$ & $1.38(1.35-1.41)$ \\
\hline \multicolumn{5}{|l|}{ Health region } \\
\hline Central & Reference & Reference & Reference & Reference \\
\hline West & $1.09(1.06-1.12)$ & $1.14(1.11-1.17)$ & $1.17(1.14-1.21)$ & $1.19(1.15-1.22)$ \\
\hline East & $1.07(1.04-1.10)$ & $1.05(1.02-1.08)$ & $1.09(1.05-1.12)$ & $1.08(1.05-1.11)$ \\
\hline North & $1.19(1.14-1.24)$ & $1.20(1.15-1.26)$ & $1.25(1.20-1.31)$ & $1.27(1.21-1.33)$ \\
\hline Toronto & $1.39(1.33-1.46)$ & $1.51(1.44-1.59)$ & $1.41(1.35-1.48)$ & $1.48(1.41-1.56)$ \\
\hline C statistic for all assessments & 0.560 & 0.617 & 0.598 & 0.609 \\
\hline $\begin{array}{l}\text { C statistic for assessments completed exclusively } \\
\text { over the phone }\end{array}$ & 0.565 & 0.622 & 0.593 & 0.604 \\
\hline
\end{tabular}




\section{Research}

Table 5: Adjusted odds ratios of emergency department visit within 90 days of interRAl CA assessment among the intake home care population

Adjusted OR $(95 \% \mathrm{Cl})^{*}$

\begin{tabular}{|c|c|c|c|}
\hline \multirow[b]{2}{*}{ Variable } & \multicolumn{3}{|c|}{ Adjusted OR $(95 \% \mathrm{Cl})^{*}$} \\
\hline & $\begin{array}{l}\text { Base model } \\
\text { (age, sex, health } \\
\text { region only) }\end{array}$ & $\begin{array}{l}\text { CHESS-CA, } \\
\text { adjusted for age, } \\
\text { sex, health region }\end{array}$ & $\begin{array}{c}\text { AUA, } \\
\text { adjusted for age, } \\
\text { sex, health region }\end{array}$ \\
\hline \multicolumn{4}{|c|}{ CHESS-CA, score } \\
\hline 0 & & Reference & \\
\hline 1 & & 0.99 (0.97-1.02) & \\
\hline 2 & & $1.14(1.11-1.16)$ & \\
\hline 3 & & $1.42(1.38-1.46)$ & \\
\hline 4 & & $1.49(1.43-1.55)$ & \\
\hline \multicolumn{4}{|l|}{5} \\
\hline \multicolumn{4}{|c|}{ AUA, score } \\
\hline 1 & & & Reference \\
\hline 2 & & & $1.54(1.48-1.60)$ \\
\hline 3 & & & $1.54(1.49-1.59)$ \\
\hline 4 & & & $1.03(1.00-1.06)$ \\
\hline 5 & & & $1.13(1.10-1.17)$ \\
\hline 6 & & & $1.24(1.20-1.28)$ \\
\hline
\end{tabular}

FI-CA, score

0-0.19

Reference

0.20-0.29

0.90 (0.87-0.92)

0.30-0.39

$0.91(0.89-0.93)$

0.40-0.49

$0.94(0.92-0.97)$

0.50-0.59

$1.02(0.99-1.05)$

$\geq 0.60$

1.06 (1.03-1.09)

Age group, yr

$\begin{array}{lcccc}18-49 & \text { Reference } & \text { Reference } & \text { Reference } & \text { Reference } \\ 50-59 & 0.98(0.95-1.02) & 0.96(0.92-0.99) & 1.00(0.96-1.03) & 0.99(0.95-1.02) \\ 60-69 & 0.90(0.87-0.93) & 0.86(0.83-0.89) & 0.93(0.90-0.96) & 0.90(0.87-0.93) \\ 70-79 & 0.81(0.78-0.83) & 0.77(0.74-0.79) & 0.85(0.82-0.88) & 0.80(0.78-0.83) \\ 80-89 & 0.70(0.68-0.72) & 0.65(0.63-0.67) & 0.74(0.72-0.76) & 0.69(0.67-0.71) \\ \geq 90 & 0.64(0.61-0.66) & 0.59(0.57-0.61) & 0.68(0.66-0.71) & 0.62(0.60-0.65)\end{array}$

Sex

Female

Male

Reference

$1.24(1.22-1.27)$

\section{Reference}

1.25 (1.23-1.27)

Reference
$1.23(1.21-1.25)$

Reference

Health region

\begin{tabular}{lc} 
Central & Reference \\
\hline West & $1.06(1.04-1.08)$ \\
\hline East & $1.13(1.10-1.15)$ \\
\hline North & $1.26(1.21-1.30)$ \\
\hline Toronto & $1.16(1.12-1.21)$ \\
C statistic for all assessments & 0.559 \\
$\begin{array}{l}\text { C statistic for assessments completed } \\
\text { exclusively over the phone }\end{array}$ & 0.565
\end{tabular}

exclusively over the phone

Reference
$1.08(1.05-1.10)$
$1.12(1.09-1.14)$
$1.26(1.22-1.30)$
$1.19(1.15-1.24)$
0.570
0.575

Reference
$1.07(1.05-1.10)$
$1.14(1.11-1.17)$
$1.26(1.22-1.31)$
$1.14(1.09-1.18)$
0.572
0.580

Note: AUA = Assessment Urgency Algorithm; CHESS-CA = Changes in Health, End-stage disease and Signs and Symptoms Scale for the Contact Assessment; Cl = confidence interval; FI-CA = Frailty Index for the Contact Assessment; interRAI CA = interRAI Contact Assessment; OR = odds ratio.

*Unless stated otherwise. 
Table 6: Adjusted odds ratios of perceived need for comprehensive geriatric assessment within 90 days among the intake home care population

Adjusted OR $(95 \% \mathrm{Cl})^{*}$

\begin{tabular}{|c|c|c|c|}
\hline \multirow[b]{2}{*}{ Variable } & \multicolumn{3}{|c|}{ Adjusted OR $(95 \% \mathrm{Cl})^{*}$} \\
\hline & $\begin{array}{l}\text { Base model } \\
\text { (age, sex, health } \\
\text { region only) }\end{array}$ & $\begin{array}{l}\text { CHESS-CA, } \\
\text { adjusted for age, } \\
\text { sex, health region }\end{array}$ & $\begin{array}{c}\text { AUA, } \\
\text { adjusted for age, } \\
\text { sex, health region }\end{array}$ \\
\hline \multicolumn{4}{|c|}{ CHESS-CA, score } \\
\hline 0 & & Reference & \\
\hline 1 & & $1.61(1.57-1.65)$ & \\
\hline 2 & & $2.64(2.57-2.72)$ & \\
\hline 3 & & $4.84(4.70-5.00)$ & \\
\hline 4 & & $18.16(17.11-19.28)$ & \\
\hline 5 & & $14.38(12.71-16.27)$ & \\
\hline \multicolumn{4}{|c|}{ AUA, score } \\
\hline 1 & & & Reference \\
\hline 2 & & & $1.68(1.60-1.77)$ \\
\hline 3 & & & $4.18(4.01-4.35)$ \\
\hline 4 & & & $6.20(5.96-6.45)$ \\
\hline 5 & & & $12.87(12.33-13.43)$ \\
\hline 6 & & & $28.27(27.11-29.49)$ \\
\hline
\end{tabular}

FI-CA, score

0-0.19

$0.20-0.29$

Reference

0.30-0.39

2.02 (1.95-2.09)

0.40-0.49

3.66 (3.55-3.77)

0.50-0.59

$7.33(7.10-7.56)$

$\geq 0.60$

13.54 (13.06-14.04)

Age group, yr

18-49

Reference

Reference

$25.23(24.21-26.28)$

50-59

1.36 (1.31-1.42)

1.18 (1.13-1.23)

Reference

Reference

60-69

1.65 (1.59-1.71)

$1.36(1.31-1.41)$

1.34 (1.28-1.40)

$1.28(1.23-1.34)$

70-79

2.54 (2.45-2.63)

$2.02(1.94-2.09)$

$1.56(1.50-1.63)$

$1.47(1.41-1.53)$

80-89

4.28 (4.14-4.43)

3.38 (3.26-3.51)

$2.10(2.02-2.19)$

$1.94(1.86-2.01)$

$\geq 90$

5.95 (5.70-6.21)

3.13 (3.02-3.26)

2.79 (2.68-2.90)

Sex

Female

Reference

4.61 (4.41-4.82)

3.84 (3.66-4.02)

3.11 (2.96-3.26)

Male

0.92 (0.91-0.94)

Reference

Reference

Reference

Health region

Central

Reference

0.91 (0.90-0.93)

0.99 (0.97-1.00)

0.96 (0.95-0.98)

West

0.67 (0.66-0.69)

Reference

Reference

Reference

East

$0.83(0.81-0.85)$

$0.69(0.68-0.71)$

0.83 (0.81-0.85)

$0.81(0.79-0.83)$

North

$0.70(0.68-0.73)$

$0.78(0.76-0.80)$

$0.84(0.82-0.86)$

$0.80(0.78-0.82)$

Toronto

$1.13(1.09-1.18)$

$0.69(0.67-0.72)$

$0.81(0.78-0.84)$

$0.80(0.77-0.83)$

C statistic for all assessments

0.659

$1.31(1.25-1.36)$

$1.30(1.24-1.36)$

$1.39(1.33-1.45)$

C statistic for assessments completed

0.655

0.733

0.791

0.796

0.735

0.781

0.785

exclusively over the phone

Note: AUA = Assessment Urgency Algorithm; CHESS-CA = Changes in Health, End-stage disease and Signs and Symptoms Scale for the Contact Assessment; Cl = confidence interval; $\mathrm{FI}-\mathrm{CA}=$ Frailty Index for the Contact Assessment; OR = odds ratio.

*Unless stated otherwise. 


\begin{tabular}{|c|c|c|c|c|c|c|c|c|c|c|c|c|}
\hline \multirow[b]{3}{*}{ Variable } & \multicolumn{12}{|c|}{$\%$ of patients; age group, $y r$; and sex } \\
\hline & \multicolumn{2}{|c|}{$18-49$} & \multicolumn{2}{|c|}{$50-59$} & \multicolumn{2}{|c|}{$60-69$} & \multicolumn{2}{|c|}{$70-79$} & \multicolumn{2}{|c|}{$80-89$} & \multicolumn{2}{|c|}{$\geq 90$} \\
\hline & Male & Female & Male & Female & Male & Female & Male & Female & Male & Female & Male & Female \\
\hline \multicolumn{13}{|c|}{ CHESS-CA, score } \\
\hline 0 & 0.6 & 0.7 & 1.3 & 1.0 & 1.9 & 1.5 & 2.3 & 1.6 & 4.1 & 2.1 & 4.1 & 4.5 \\
\hline 1 & 1.8 & 1.1 & 2.3 & 2.0 & 3.2 & 1.9 & 3.8 & 2.7 & 5.3 & 3.3 & 8.3 & 5.4 \\
\hline 2 & 2.7 & 1.8 & 4.9 & 3.5 & 6.4 & 4.2 & 7.6 & 4.3 & 9.0 & 5.2 & 12.2 & 7.5 \\
\hline 3 & 9.5 & 6.5 & 11.3 & 9.9 & 14.8 & 9.6 & 16.4 & 9.5 & 15.9 & 9.3 & 19.2 & 13.3 \\
\hline 4 & 35.4 & 37.9 & 38.7 & 31.3 & 42.7 & 32.6 & 35.9 & 27.9 & 32.1 & 19.7 & 33.7 & 22.5 \\
\hline 5 & 61.9 & 57.8 & 71.4 & 52.5 & 62.7 & 59.4 & 61.3 & 57.1 & 56.2 & 52.9 & 62.1 & 45.2 \\
\hline \multicolumn{13}{|l|}{ AUA, score } \\
\hline 1 & 0.6 & 0.5 & 1.4 & 1.0 & 1.5 & 0.6 & 2.1 & 1.3 & 3.6 & 2.3 & 5.5 & 2.9 \\
\hline 2 & 1.1 & 0.9 & 2.9 & 2.3 & 4.1 & 2.4 & 3.5 & 3.3 & 5.0 & 2.8 & 8.8 & 3.6 \\
\hline 3 & 4.1 & 2.7 & 6.2 & 5.2 & 8.8 & 5.5 & 9.6 & 6.1 & 9.5 & 4.6 & 9.0 & 6.3 \\
\hline 4 & 2.5 & 2.2 & 4.7 & 3.5 & 6.6 & 4.3 & 7.2 & 4.1 & 8.2 & 4.6 & 9.9 & 5.0 \\
\hline 5 & 3.9 & 4.2 & 9.2 & 7.8 & 14.2 & 9.5 & 15.7 & 9.7 & 15.5 & 9.9 & 18.7 & 13.2 \\
\hline 6 & 8.0 & 7.1 & 13.3 & 9.9 & 16.5 & 11.0 & 16.9 & 9.9 & 15.9 & 8.7 & 20.1 & 12.8 \\
\hline \multicolumn{13}{|l|}{ FI-CA, score } \\
\hline $0-0.19$ & 0.8 & 0.8 & 1.9 & 1.5 & 2.4 & 1.5 & 2.7 & 2.1 & 4.4 & 2.0 & 5.9 & 2.2 \\
\hline $0.20-0.29$ & 2.2 & 1.2 & 3.4 & 3.0 & 4.2 & 2.6 & 4.6 & 3.0 & 5.7 & 3.1 & 5.2 & 5.2 \\
\hline $0.30-0.39$ & 3.0 & 1.9 & 5.1 & 3.7 & 7.5 & 4.3 & 7.7 & 3.9 & 7.9 & 4.3 & 9.6 & 4.6 \\
\hline $0.40-0.49$ & 4.2 & 3.5 & 7.8 & 5.6 & 11.7 & 5.8 & 11.1 & 5.8 & 10.2 & 5.2 & 11.9 & 7.3 \\
\hline $0.50-0.59$ & 6.5 & 6.7 & 10.9 & 8.5 & 13.6 & 10.9 & 14.9 & 9.6 & 13.8 & 8.0 & 15.7 & 10.0 \\
\hline$\geq 0.60$ & 16.2 & 15.7 & 26.3 & 20.9 & 28.9 & 20.8 & 25.0 & 16.9 & 21.8 & 14.3 & 26.5 & 17.4 \\
\hline
\end{tabular}

At the time of writing, both CHESS-CA and AUA are approved interRAI algorithms and can be readily integrated into any interRAI CA implementation. In the interest of using existing decision-support tools, this study provides evidence that use of CHESS-CA and AUA together would give a clear picture about the patient's frailty status. CHESS-CA is a strong predictor of death and should prompt actions that might reduce the risk, such as expediting referrals to a physician or medical team, where appropriate. AUA is better suited for identifying patients requiring CGA (i.e., interRAI $\mathrm{HC}$ assessment), especially among patients with chronic or complex needs.

Rather than recommending a single frailty measure for which there is no overwhelming evidence, this study's findings reinforce the use of multiple algorithms as part of the "care planning toolkit." ${ }^{36}$ Care coordinators review the areas of concern identified by the algorithms and, applying their clinical expertise, develop a care plan grounded in the patient and family's needs and goals. This approach is consistent with the notion that frailty likely arises from multiple etiologies, and as such, effective management requires a team of health professionals and an individualized approach to care. ${ }^{37,38}$

In the current COVID-19 pandemic environment, CHESS-CA and AUA combined with syndromic and exposure risk assessment could identify vulnerable people who may benefit from targeted proactive case finding and remote monitoring across primary, acute and continuing care settings. Based on current knowledge that frailty is an important risk factor for severe COVID-19, individuals and their families and their circle of care may be better prepared to engage in goals of care or end-of-life discussions. ${ }^{39}$ As a result, early in April 2020, interRAI members from Canada, South Africa and Finland developed a brief self-reported instrument called the interRAI COVID-19 Vulnerability Screener (CVS) that calculates AUA among other outputs. ${ }^{40}$ Future research should evaluate the association between AUA and adverse outcomes related to COVID-19 disease among those assessed with either the interRAI CA or interRAI CVS. 


\begin{tabular}{|c|c|c|c|c|c|c|c|c|c|c|c|c|}
\hline \multirow[b]{3}{*}{ Variable } & \multicolumn{12}{|c|}{$\%$ of patients; age group, yr; and sex } \\
\hline & \multicolumn{2}{|c|}{$18-49$} & \multicolumn{2}{|c|}{$50-59$} & \multicolumn{2}{|c|}{$60-69$} & \multicolumn{2}{|c|}{$70-79$} & \multicolumn{2}{|c|}{$80-89$} & \multicolumn{2}{|c|}{$\geq 90$} \\
\hline & Male & Female & Male & Female & Male & Female & Male & Female & Male & Female & Male & Female \\
\hline \multicolumn{13}{|c|}{ CHESS-CA, score } \\
\hline 0 & 9.6 & 9.4 & 10.0 & 9.2 & 11.1 & 9.1 & 12.4 & 8.4 & 14.6 & 10.6 & 14.9 & 13.5 \\
\hline 1 & 10.9 & 11.1 & 14.1 & 11.1 & 14.0 & 10.1 & 15.8 & 11.7 & 17.4 & 12.8 & 20.6 & 14.9 \\
\hline 2 & 15.3 & 13.9 & 17.1 & 13.5 & 19.1 & 14.5 & 20.5 & 14.6 & 22.0 & 15.5 & 24.4 & 17.3 \\
\hline 3 & 24.0 & 20.7 & 24.6 & 21.4 & 26.3 & 21.5 & 28.3 & 21.6 & 26.6 & 19.4 & 27.4 & 21.9 \\
\hline 4 & 36.5 & 37.9 & 35.6 & 35.0 & 35.5 & 30.4 & 31.2 & 28.3 & 29.5 & 22.8 & 24.6 & 22.5 \\
\hline 5 & 28.6 & 35.6 & 27.5 & 24.8 & 26.7 & 32.1 & 25.8 & 26.0 & 18.8 & 15.8 & 16.4 & 11.9 \\
\hline \multicolumn{13}{|l|}{ AUA, score } \\
\hline 1 & 8.4 & 8.9 & 8.9 & 7.2 & 9.1 & 7.4 & 11.1 & 7.3 & 13.0 & 9.5 & 14.4 & 10.8 \\
\hline 2 & 13.2 & 11.4 & 13.4 & 11.2 & 15.1 & 11.7 & 15.4 & 11.5 & 17.1 & 11.4 & 12.9 & 13.0 \\
\hline 3 & 16.4 & 15.7 & 19.6 & 15.0 & 21.0 & 15.6 & 21.5 & 15.5 & 20.2 & 13.0 & 21.3 & 12.5 \\
\hline 4 & 12.3 & 11.0 & 14.3 & 13.1 & 15.7 & 12.9 & 18.1 & 13.5 & 19.7 & 14.1 & 22.5 & 15.8 \\
\hline 5 & 10.9 & 14.0 & 18.1 & 17.8 & 21.3 & 17.1 & 23.9 & 18.6 & 24.0 & 18.7 & 22.6 & 19.1 \\
\hline 6 & 16.7 & 16.0 & 23.3 & 19.2 & 26.0 & 20.0 & 25.8 & 19.5 & 24.8 & 17.8 & 26.8 & 20.4 \\
\hline \multicolumn{13}{|l|}{ Fl-CA, score } \\
\hline $0-0.19$ & 10.2 & 9.9 & 11.6 & 9.6 & 11.8 & 9.7 & 12.9 & 9.3 & 15.2 & 9.9 & 16.9 & 10.3 \\
\hline $0.20-0.29$ & 13.4 & 12.2 & 13.9 & 10.3 & 15.0 & 10.9 & 16.1 & 11.2 & 17.0 & 12.3 & 16.5 & 14.4 \\
\hline $0.30-0.39$ & 14.5 & 13.1 & 17.1 & 13.6 & 18.3 & 13.0 & 19.8 & 14.2 & 18.9 & 13.3 & 22.0 & 14.9 \\
\hline $0.40-0.49$ & 15.1 & 14.4 & 20.7 & 15.6 & 23.1 & 16.1 & 22.6 & 15.9 & 22.0 & 15.5 & 22.9 & 18.2 \\
\hline $0.50-0.59$ & 15.0 & 19.0 & 21.4 & 23.4 & 24.4 & 21.8 & 25.9 & 19.5 & 24.6 & 17.8 & 24.9 & 18.0 \\
\hline$\geq 0.60$ & 21.1 & 20.8 & 28.9 & 25.5 & 29.7 & 24.9 & 28.3 & 24.0 & 27.1 & 21.3 & 26.6 & 21.5 \\
\hline $\begin{array}{l}\text { Note: } A \cup A=A s \\
\text { FI-CA = Frailty } \\
{ }^{*} \text { The shade of } \\
\text { darkest shade }\end{array}$ & $\begin{array}{l}\text { int } \\
\text { in th } \\
\text { the } \\
\text { the }\end{array}$ & $\begin{array}{l}\text { Algoritl } \\
\text { ct Asse } \\
\text { dicates } \\
\text { oserver }\end{array}$ & $\begin{array}{l}\text { HES } \\
\text { ht; in } \\
\text { bse }\end{array}$ & $\begin{array}{l}\text { Char } \\
\text { CA = } \\
\text { pporti }\end{array}$ & II Co & $\begin{array}{l}\text {-stage } \\
\text { ssess } \\
\text { ssion }\end{array}$ & an & and & ns 5 & or the & As & \\
\hline
\end{tabular}

\section{Limitations}

Compared with other studies focused on Ontario long-stay home care patients, our study includes all home care patients in the province, contributing to its generalizability. Patientlevel linkage of HCRS, DAD and NACRS databases based on unique patient identifiers and exact assessment and event dates enables us to have high confidence in the rates of hospital admissions and emergency department visits.

Several limitations should be noted. In the present study, we created the FI-CA for use as the reference standard for frailty measurement based on the accumulation of deficits principle. Although the FI-CA made it possible to directly compare the results from a widely accepted conceptualization of frailty with other algorithms, it is important to note that the FI-CA itself has not been tested or validated beyond this study. Second, deaths were identified from the discharge disposition found in home care and hospital administrative records. If the patient moved to another setting (e.g., long-term care facility) and died in that setting within the 90-day period, the discharge dispo- sition would not be revised retroactively, resulting in under-reporting of actual deaths. Nevertheless, the AUC statistics and relative differences in the odds ratios for death between the frailty levels were consistent with those of previous studies.

Third, although the interRAI CA is completed by a trained health professional, some questions, such as changes in cognition or activities of daily living status in the last 90 days, are prone to self-report and recall biases. The presence of these biases reinforce the importance of using the full "care planning toolkit" and viewing the patient's health status as a whole rather than relying on a single indicator.

\section{Conclusion}

Frailty measures (CHESS-CA, AUA, FI-CA) from the interRAI CA identified patients at higher risk for death and hospital admission within 90 days and those perceived to benefit from comprehensive follow-up assessment. The frailty measures added little to the prediction of emergency department visits. CHESS-CA and AUA are standard algorithms already 


\begin{tabular}{|c|c|c|c|c|c|c|c|c|c|c|c|c|}
\hline \multirow[b]{3}{*}{ Variable } & \multicolumn{12}{|c|}{$\%$ of patients; age group, yr; and sex } \\
\hline & \multicolumn{2}{|c|}{$18-49$} & \multicolumn{2}{|c|}{$50-59$} & \multicolumn{2}{|c|}{$60-69$} & \multicolumn{2}{|c|}{$70-79$} & \multicolumn{2}{|c|}{$80-89$} & \multicolumn{2}{|c|}{$\geq 90$} \\
\hline & Male & Female & Male & Female & Male & Female & Male & Female & Male & Female & Male & Female \\
\hline \multicolumn{13}{|c|}{ CHESS-CA, score } \\
\hline 0 & 18.4 & 19.7 & 20.7 & 20.8 & 21.7 & 21.4 & 27.1 & 29.5 & 38.1 & 41.9 & 45.2 & 48.9 \\
\hline 1 & 24.5 & 25.2 & 25.8 & 27.3 & 29.0 & 30.6 & 36.5 & 42.4 & 50.6 & 55.1 & 60.3 & 63.2 \\
\hline 2 & 31.7 & 34.8 & 36.6 & 39.3 & 39.0 & 42.1 & 50.7 & 52.7 & 64.7 & 66.4 & 72.6 & 73.2 \\
\hline 3 & 46.2 & 48.0 & 53.2 & 52.7 & 57.4 & 59.4 & 67.0 & 67.3 & 76.3 & 76.1 & 82.2 & 82.0 \\
\hline 4 & 83.7 & 83.8 & 87.9 & 87.0 & 89.1 & 89.3 & 89.1 & 89.2 & 90.5 & 89.6 & 88.1 & 87.0 \\
\hline 5 & 85.7 & 75.6 & 89.0 & 85.2 & 88.9 & 89.1 & 86.3 & 85.8 & 85.6 & 84.5 & 86.2 & 83.6 \\
\hline \multicolumn{13}{|l|}{ AUA, score } \\
\hline 1 & 8.4 & 9.2 & 10.1 & 9.9 & 9.1 & 8.3 & 11.2 & 11.2 & 18.9 & 18.3 & 27.8 & 23.4 \\
\hline 2 & 11.9 & 13.7 & 16.3 & 14.9 & 16.4 & 17.0 & 18.8 & 20.5 & 22.3 & 25.2 & 31.3 & 28.8 \\
\hline 3 & 29.5 & 27.8 & 34.9 & 32.8 & 36.1 & 34.5 & 37.2 & 36.2 & 42.5 & 40.5 & 48.1 & 44.1 \\
\hline 4 & 27.4 & 28.8 & 33.3 & 33.5 & 36.8 & 38.8 & 45.1 & 49.7 & 58.2 & 61.9 & 67.2 & 67.8 \\
\hline 5 & 36.3 & 42.9 & 48.8 & 53.7 & 60.8 & 61.4 & 69.6 & 69.3 & 75.7 & 75.1 & 74.9 & 73.3 \\
\hline 6 & 63.5 & 63.6 & 70.7 & 69.2 & 76.5 & 74.5 & 83.4 & 81.6 & 87.8 & 86.4 & 89.9 & 89.3 \\
\hline \multicolumn{13}{|l|}{ FI-CA, score } \\
\hline $0-0.19$ & 12.5 & 13.3 & 15.1 & 14.7 & 14.6 & 15.1 & 16.3 & 18.0 & 22.9 & 25.1 & 27.4 & 32.4 \\
\hline $0.20-0.29$ & 21.5 & 22.6 & 25.0 & 23.0 & 25.4 & 25.7 & 30.0 & 31.9 & 38.1 & 41.3 & 46.0 & 48.0 \\
\hline $0.30-0.39$ & 30.5 & 30.2 & 36.1 & 35.0 & 38.9 & 37.3 & 43.5 & 46.5 & 54.2 & 57.0 & 62.4 & 62.7 \\
\hline $0.40-0.49$ & 41.9 & 45.3 & 52.3 & 51.2 & 57.0 & 55.0 & 63.0 & 63.2 & 71.1 & 73.2 & 75.8 & 73.3 \\
\hline $0.50-0.59$ & 52.9 & 59.4 & 63.9 & 67.4 & 71.7 & 74.3 & 77.3 & 79.6 & 81.7 & 81.5 & 82.5 & 79.5 \\
\hline$\geq 0.60$ & 71.4 & 72.8 & 79.4 & 79.3 & 84.7 & 84.4 & 88.7 & 87.8 & 90.1 & 88.1 & 87.5 & 86.6 \\
\hline
\end{tabular}

embedded into the interRAI CA that offer decision support within existing systems. These measures offer quick identification of frail home care patients at admission (and the general population that could be assessed with the interRAI CVS) who face increased risk during regular times but are particularly vulnerable during a disease outbreak.

\section{References}

1. Portraits of home care in Canada. Mississauga (ON): Canadian Home Care Association; 2013.

2. Chappell NL, Dlitt BH, Hollander MJ, et al. Comparative costs of home care and residential care. Gerontologist 2004;44:389-400.

3. Caplan GA, Sulaiman NS, Mangin DA, et al. A meta-analysis of "hospital in the home." In: Database of Abstracts of Reviews of Effects (DARE): Quality-Assessed Reviews. York (UK): Centre for Reviews and Dissemination; 2012. Available: www.ncbi.nlm.nih.gov/books/NBK127330/ (accessed 2020 Apr. 13).

4. Forder J. Long-term care and hospital utilisation by older people: an analysis of substitution rates. Health Econ 2009;18:1322-38.

5. Clegg A, Young J, Iliffe S, et al. Frailty in elderly people. Lancet 2013;381:752-62.

6. Fried LP, Ferrucci L, Darer J, et al. Untangling the concepts of disability, frailty, and comorbidity: implications for improved targeting and care. $\mathcal{F}$ Gerontol A Biol Sci Med Sci 2004;59:255-63.
7. Maxwell CJ, Campitelli MA, Diong C, et al. Variation in the health outcomes associated with frailty among home care clients: relevance of caregiver distress and client sex. BMC Geriatr 2018;18:211.

8. Landi F, Barillaro C, Bellieni A, et al. The new challenge of geriatrics: saving frail older people from the SARS-CoV-2 pandemic infection. 7 Nutr Health Aging 2020;24:466-70.

9. Garnier-Crussard A, Forestier E, Gilbert T, et al. Novel coronavirus (COVID-19) epidemic: What are the risks for older patients? 7 Am Geriatr Soc 2020;68:939-40.

10. Abbatecola AM, Antonelli-Incalzi R. COVID-19 spiraling of frailty in older Italian patients. 7 Nutr Health Aging 2020;24:453-5.

11. Sinclair AJ, Abdelhafiz AH. Age, frailty and diabetes - triple jeopardy for vulnerability to COVID-19 infection. EClinicalMedicine 2020;22:100343.

12. Hirdes JP, Curtin-Telegdi N, Poss JW, et al. interRAI Contact Assessment (CA) form and user's manual: a screening level assessment for emergency department and intake from community/hospital. Version 9.2. Rockport (MA): interRAI; 2010.

13. Morris JN, Fries BE, Bernabei R, et al. interRAI Home Care (HC) assessment form and user's manual. Version 9.1, Canadian Edition. Washington (DC): interRAI; 2012.

14. Morris JN, Howard EP, Steel KR. Development of the interRAI home care frailty scale. BMC Geriatr 2016;16:188.

15. Hirdes JP, Poss JW, Mitchell L, et al. Use of the interRAI CHESS scale to predict mortality among persons with neurological conditions in three care settings. PLoS One 2014;9:e99066.

16. Armstrong JJ, Stolee P, Hirdes JP, et al. Examining three frailty conceptualizations in their ability to predict negative outcomes for home-care clients. Age Ageing 2010;39:755-8. 
17. Burn R, Hubbard RE, Scrase RJ, et al. A frailty index derived from a standardized comprehensive geriatric assessment predicts mortality and aged residential care admission. BMC Geriatr 2018;18:319.

18. Hirdes JP, Ljunggren G, Morris JN, et al. Reliability of the interRAI suite of assessment instruments: a 12-country study of an integrated health information system. BMC Health Serv Res 2008;8:277.

19. Hogeveen SE, Chen J, Hirdes JP. Evaluation of data quality of interRAI assessments in home and community care. BMC Med Inform Decis Mak 2017;17:150

20. Foebel AD, Hirdes JP, Heckman GA, et al. ideas PNC research team. Diagnostic data for neurological conditions in interRAI assessments in home care, nursing home and mental health care settings: a validity study. BMC Health Serv Res 2013;13:457.

21. Poss JW, Jutan NM, Hirdes JP, et al. A review of evidence on the reliability and validity of Minimum Data Set data. Healthc Manage Forum 2008;21:33-9.

22. Landi F, Tua E, Onder G, et al.; SILVERNET-HC Study Group of Bergamo. Minimum Data Set for home care: a valid instrument to assess frail older people living in the community. Med Care 2000;38:1184-90.

23. Hirdes JP, Frijters DH, Teare GF. The MDS-CHESS scale: a new measure to predict mortality in institutionalized older people. 7 Am Geriatr Soc 2003;51:96-100.

24. Dolenc E, Rotar-Pavlič D. Frailty assessment scales for the elderly and their application in primary care: a systematic literature review. $Z d r$ Varst 2019;58:91-100.

25. Muscedere J, Andrew MK, Bagshaw SM, et al.; Canadian Frailty Network (CFN). Screening for frailty in Canada's health care system: a time for action. Can 7 Aging 2016;35:281-97.

26. Lee H, Lee E, Jang I-Y. Frailty and comprehensive geriatric assessment. 7 Korean Med Sci 2020;35:e16.

27. Brousseau A-A, Dent E, Hubbard R, et al. Identification of older adults with frailty in the emergency department using a frailty index: results from a multinational study. Age Ageing 2018;47:242-8.

28. Searle SD, Mitnitski A, Gahbauer EA, Gill TM, Rockwood K. A standard procedure for creating a frailty index. BMC Geriatr. 2008;8:24.

29. Rockwood K, Song X, Mitnitski A. Changes in relative fitness and frailty across the adult lifespan: evidence from the Canadian National Population Health Survey. CMA7 2011;183:E487-94.

30. Romero-Ortuno R. An alternative method for Frailty Index cut-off points to define frailty categories. Eur Geriatr Med 2013;4:299-303. doi: 10.1016/j. eurger.2013.06.005.

31. Campitelli MA, Bronskill SE, Hogan DB, et al. The prevalence and health consequences of frailty in a population-based older home care cohort: a comparison of different measures. BMC Geriatr 2016;16:133.

32. Scaccabarozzi G, Palmer K, Onder G, et al. Predicting care intensity in geriatric home care patients: a comparison of different measures. Aging Clin Exp Res 2020;32:281-7.

33. Au A, Puts MTE, Fletcher JD, et al. Frailty markers predicting emergency department visits in a community-dwelling sample of vulnerable seniors in Montreal. Can 7 Aging 2011;30:647-55.

34. Puts MTE, Monette J, Girre V, et al. Does frailty predict hospitalization, emergency department visits, and visits to the general practitioner in older newly-diagnosed cancer patients? Results of a prospective pilot study. Crit Rev Oncol Hematol 2010;76:142-51.

35. Costa AP, Hirdes JP, Bell CM, et al. Derivation and validation of the detection of indicators and vulnerabilities for emergency room trips scale for classifying the risk of emergency department use in frail community-dwelling older adults. $7 \mathrm{Am}$ Geriatr Soc 2015;63:763-9.

36. McMullan J, Sultan H, Haratsidis E, et al. Modernizing assessment for Ontario's home care patients using the interRAI home care. Canadian interRAI Confer- ence: Wellness for Life - A Priority for All; 2018 May 16; Toronto. Available: https://acutenet.com/wp-content/uploads/2018/06/C1.1-Assessment-Modernization interRAI-Conference-Final_McMullan.pdf(accessed 2020 June 16).

37. Chen X, Mao G, Leng SX. Frailty syndrome: an overview. Clin Interv Aging 2014;9:433-41.

38. Walston J, Buta B, Xue Q-L. Frailty screening and interventions: considerations for clinical practice. Clin Geriatr Med 2018;34:25-38.

39. Pattison N. End-of-life decisions and care in the midst of a global coronavirus (COVID-19) pandemic. Intensive Crit Care Nurs 2020;58:102862.

40. Hirdes JP, Heckman G, Geffen L, Saari M, Hogeveen S, Northwood M. interRAI COVID-19 Vulnerability Screener (CVS) User Guide. Washington (DC): interRAI; in press.

Affiliations: School of Public Health and Health Systems (Sinn, Heckman, Poss, Hirdes), University of Waterloo; Research Institute for Aging (Heckman), Waterloo, Ont.; Department of Cardiovascular, Metabolic and Aging Diseases (Onder), Istituto Superiore di Sanità, Rome, Italy; Fondazione Policlinico Universitario Agostino Gemelli IRCCS (Vetrano), Università Cattolica del Sacro Cuore, Rome, Italy; Aging Research Center (Vetrano), Department of Neurobiology Care Sciences and Society, Karolinska Institutet, Stockholm, Sweden

Contributors: Chi-Ling Sinn contributed to conceptualization, data curation, formal analysis, methodology, validation, writing the original draft and reviewing and editing. George Heckman contributed to conceptualization, funding acquisition, and reviewing and editing. Jeffrey Poss contributed to data curation, formal analysis, methodology, and reviewing and editing. Graziano Onder and Davide Vetrano contributed to validation and reviewing and editing. John Hirdes contributed to conceptualization, funding acquisition, resources, supervision, and reviewing and editing. All authors gave final approval of the version to be published and agreed to be accountable for all aspects of the work.

Funding: This research is funded by the Canadian Frailty Network (previously known as the Technology Evaluation in the Elderly Network), which is supported by the Government of Canada through the Networks of Centres of Excellence program.

Data sharing: While a data sharing agreement between the Canadian Institute for Health Information (CIHI) and the University of Waterloo does not allow for the study authors to publish the data set publicly, the databases used in this study are available on reasonable request from CIHI or through collaboration on a research project directly with interRAI Canada.

Acknowledgements: We gratefully acknowledge and thank Andrew P. Costa $\mathrm{PhD}$ (Department of Medicine, McMaster University, Hamilton, Ontario, Canada) and Paul C. Hébert MD MHSc MA (Département de Médecine, Université de Montréal et Centre hospitalier de l'Université de Montréal, Montréal, Québec, Canada) for reviewing and editing earlier drafts of the manuscript.

Supplemental information: For reviewer comments and the original submission of this manuscript, please see www.cmajopen.ca/content/8/4/ E796/suppl/DC1. 